\title{
Randomized Trial of Sternal Closure for Low Risk Patients: Rigid Fixation versus Wire Closure
}

\author{
Graham Peigh, MD, Jeevan Kumar, MD, Shinya Unai, MD, James T. Diehl, MD, Hitoshi Hirose, MD, PhD \\ Division of Cardiothoracic Surgery, Thomas Jefferson University Hospital, Philadelphia, Pennsylvania, USA
}

\section{ABSTRACT}

Background: A previous retrospective analysis of our cardiac surgery patients showed shortened ventilation time and hospital stay among patients receiving rigid sternal fixation compared to sternal wire fixation. We performed a prospective randomized study to further investigate these outcomes and determine if rigid closure can provide reduced pain after cardiac surgery.

Methods: Patients undergoing cardiac surgery between July 2011 and May 2014 were prospectively randomized into wire closure (Group C) or rigid fixation using sternal plates (Group R) groups. Age above 80, emergency surgery, redo sternotomy, and immunosuppression were among major exclusion criteria precluding randomization. Intubation time was recorded for all patients. Pain scores were determined daily from postoperative day 1 until day 5 at 6 a.m. using a numeric rating scale. Narcotic pain medication requirements from day 1 to 5 were collected and converted into intravenous morphine equivalents.

Results: Of 80 patients, 39 patients were in Group R (average age $65 \pm 8 ; 31$ male and 8 female) and 41 patients were in Group C (average age $66 \pm 9 ; 34$ male and 7 female). Group $\mathrm{R}$ patients had a higher body mass index than patients in Group C (Group R: $31 \pm 5$; Group C: $29 \pm 5$; $P=.04)$. No significant differences in the end points of intubation time and postoperative pain were observed.

Conclusion: This randomized study of cardiac surgery patients showed no significant benefits of rigid fixation over conventional sternal wire closure with regard to intubation time, postoperative pain, or length of hospital stay.

\section{INTRODUCTION}

Despite advances in cardiothoracic surgical technique, the majority of open-heart surgeries are performed via a median sternotomy [Mozaffarian 2016]. Since the introduction of

Received Fune 24, 2017; received in revised form August 1, 2017; accepted August 1, 2017.

This research was supported with a research grant between Biomet and Thomas Fefferson University (grant \#B101993.1) and approved by IRB (approval \#10C.474). Biomet provided an educational grant to Thomas Fefferson University for this study.

Correspondence: Hitoshi Hirose, MD, Division of Cardiothoracic Surgery, Thomas Fefferson University, 1025 Walnut Street, Room 605, Philadelphia, PA 19107; 215-955-5654, fax: 215-955-6010 (e-mail: Hitoshi.Hirose@jefferson.edu). cardiac surgery, sternal wires have been the gold standard to re-approximate the sternum. Wire closure is procedurally simple and usually provides enough stability for the sternum. However, sternal wire closure does not always completely secure the sternum following sternotomy, as the sternum secured by wires may continue to move with normal respiratory motions [Ozaki 1998; Hirose 2011]. Rather than relying on sternal wires, research has investigated the benefits of rigid sternal closure devices using plate and screws. As previously reported, these rigid devices allow for three-dimensional support on the reapproximated sternum rather than the twopoint support offered by conventional wire. Cadaveric studies have affirmed this by showing that rigid sternal fixation is associated with less lateral movement and enhanced sternal stiffness as compared to wire fixation [Ozaki 1998]. Decreases in lateral movement with rigid sternal fixation were further shown in an in vitro study of sternotomy patients [Pai 2005]. Reduced sternal separation and improved sternal healing have also been shown in clinical studies [Raman 2012; Allen 2017; Nishimura 2014]. Because better sternum stabilization is achieved by rigid closure, patients who undergo rigid closure may have the benefits of better pain control, early mobilization and early discharge from the hospital. Two prospective randomized multicenter trials have compared rigid sternal fixation with wire cerclage following a median sternotomy, and have shown significant improvements in sternal healing, reduced postoperative pain, and fewer sternal complications. In addition, the most recent trial showed rigid plate fixation was not associated with higher healthcare costs through six months [Raman 2012; Allen 2017].

Our previous retrospective study found that among patients undergoing sternotomy, rigid sternal fixation was associated with shorter ICU time and postoperative length of stay. However, the patients who received rigid sternal fixation were shown to have a lower risk profile [Hirose 2011]. Based on these results, it was determined that patient randomization was necessary to evaluate the true efficacy of rigid closure with respect to these endpoints. The present study is a randomized trial to further investigate these outcomes and to determine if rigid closure can provide shorter intubation times, better immediate pain outcomes, and shorter ICU and hospital stays after cardiac surgery in relatively low risk patients. In contrast to previous studies, we sought to assess short-term (rather than intermediate or long-term) pain in patients who undergo rigid and wire sternal fixation. In addition, previous studies on methods of sternal fixation have focused on highrisk patient populations [Song 2004]. Unique to this study is the enrollment of non-high risk patients for randomization. 


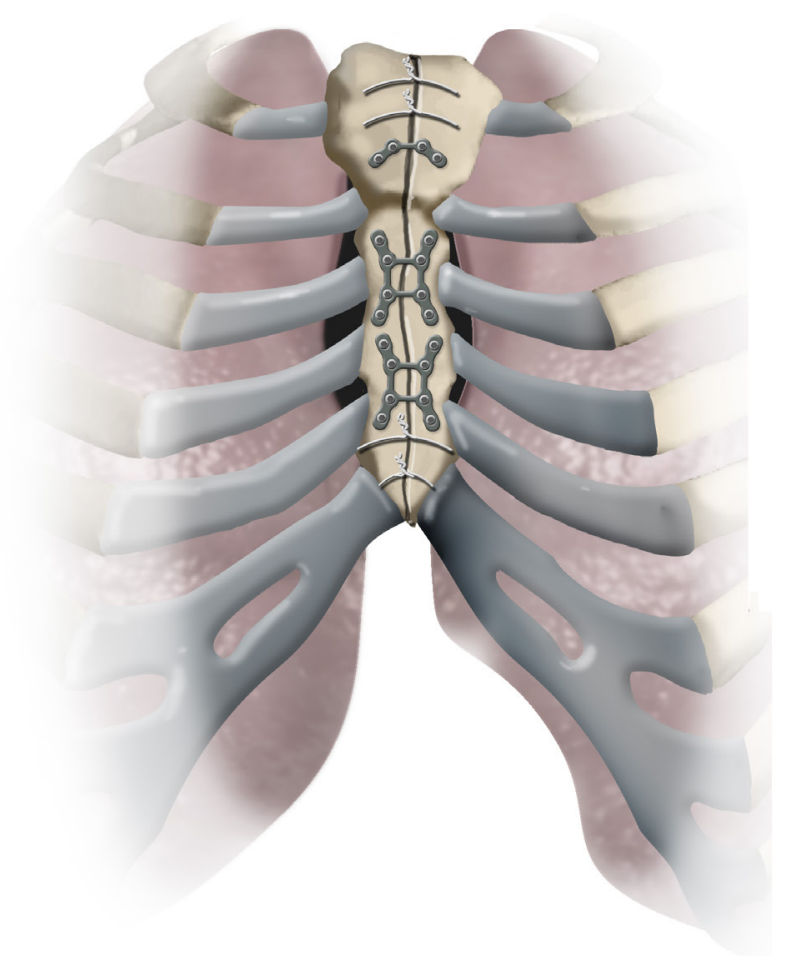

Figure 1. Example of rigid closure (left) and wire closure (right).

\section{MATERIALS AND METHODS}

All patients undergoing a coronary artery bypass grafting (CABG) procedure and/or a cardiac valve procedure between July 2011 and May 2014 were evaluated for study eligibility. If eligible, patients were randomized into a conventional wire (Group C) or rigid sternal fixation (Group R) group.

Rigid sternal fixation was accomplished by using the SternalockBlu Closure System (Biomet, Jacksonville, Florida, USA). A description of the closure technique has been published elsewhere, and by our group [Hirose 2011; Raman 2007]. Our study was supported by an educational grant from Biomet (Grant \#B101993.1). Wire sternal closure was achieved with stainless steel wires (Ethicon, San Antonio, Texas, USA) using a standard single cerclage technique [Hirose 2011] (Figure 1). After sternal closure, the subxiphoid fascia was closed with interrupted sutures using 0 -Vicyl (Ethicon, Somerville, NJ, USA) and sternal fascia was closed with continuous sutures using 0 -Vicyl. Subcutaneous tissue was reapproximated with continuous 3-0 Vicryl and skin was closed with 4-0 Monocryl (Ethicon, Somerville, NJ, USA).

In this parallel group study, randomization was roughly 1:1. Exclusions from randomization included being over 80 years old, requiring emergent surgery, a redo sternotomy, an intraoperative finding of osteoporosis, increased complexity of the surgery as determined by the surgeon, or the presence of renal failure, immunosuppression, infection, or malignancy. Minimally invasive surgeries, off-pump surgeries, thoracotomy approach, and robotic procedures were also excluded. A complete list of exclusion criteria is shown in

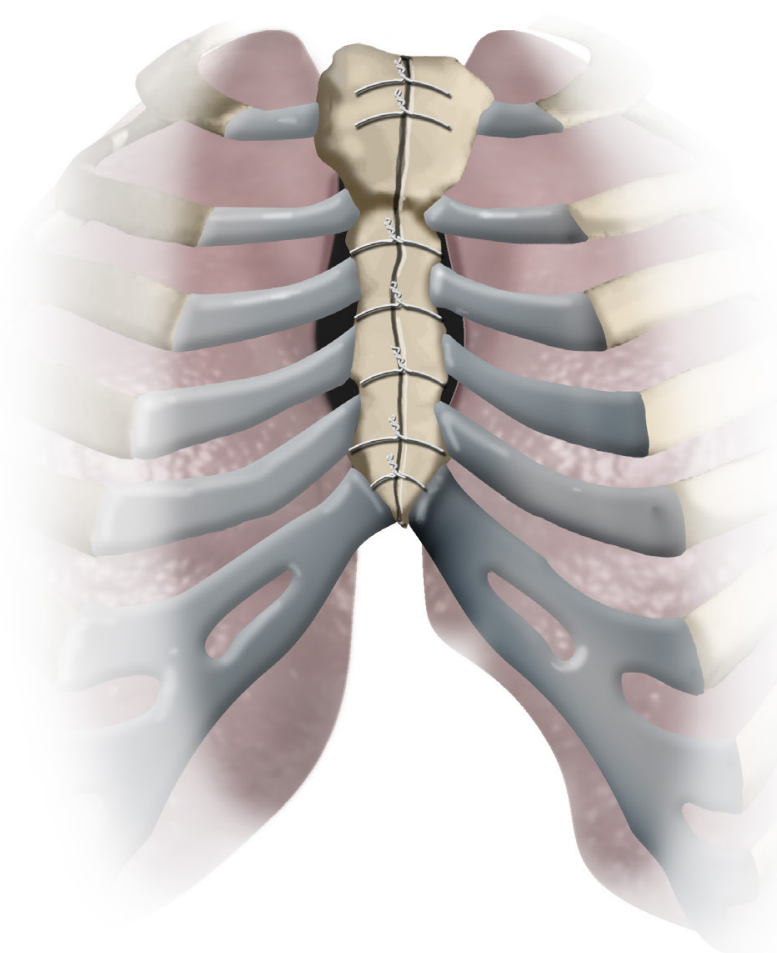

Figure 2. Only a select number of surgeons were included in this trial, as to maintain homogeneity.

This study was initially powered based on a projected sample size of 400 patients. Due to difficulty in enrollment and the change of our postoperative pain control procedure, our study was discontinued after enrollment of 80 patients.

All data was obtained from surgeries performed at Thomas Jefferson University Hospital in Philadelphia, Pennsylvania. No changes to the methods were made after trial commencement. Randomization was initiated after approval of Institutional Review Board (IRB approval \#10C.474) and written consent was obtained preoperatively from each patient who participated in the study. The study protocol was uploaded to ClincialTrial. gov (ClinicalTrial.gov ID \#NCT 01317095) for transparency.

Prior to surgery, patients were interviewed and consented for the study. If the surgeon believed that intraoperative exclusions such as unexpected osteoporosis, major bleeding, a complex surgery, the usage of bilateral mammary arteries, or unavailability of the SternalockBlu device were met, randomization was not done and the patient's sternum was reapproximated with conventional sternal wire. After appropriate hemostasis for the procedure was achieved, randomization was performed by opening sealed envelopes that contained a 1:1 ratio of randomization cards.

The primary endpoint for this study was intubation time. Secondary endpoints were postoperative pain, ICU stay length, and length of total hospitalization. We also monitored for differences in rates of sternal infections, device related complications, and the incidence of pneumonia. All outcomes were analyzed upon completion of enrollment. 


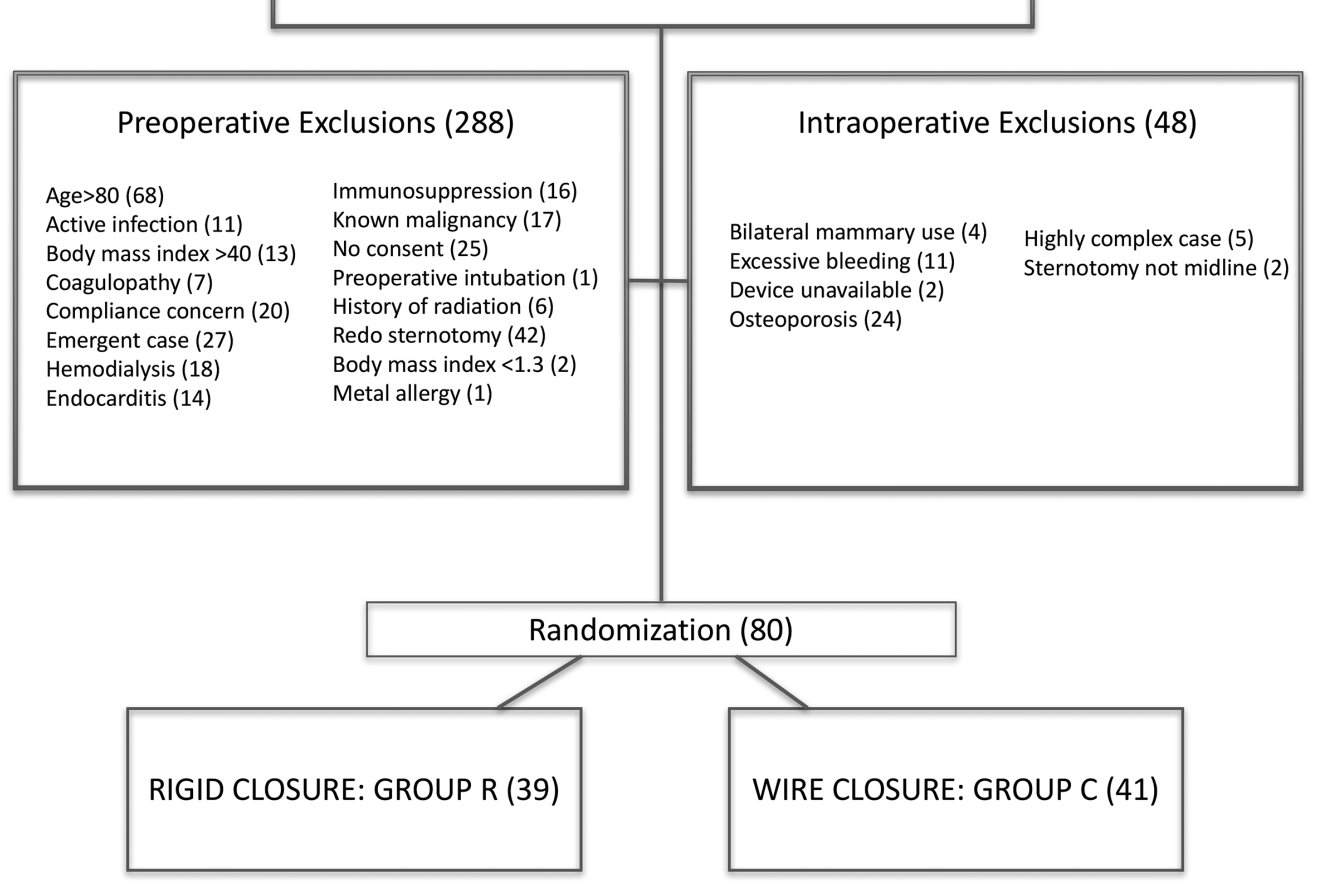

Figure 2. Exclusion criteria and randomization.

On postoperative days 1-5, patients were asked to rate their pain at 6 a.m. on a Likert Scale from 1-10 (0: no pain; 10: worst possible pain). Narcotic pain medication usage was also recorded for patients from postoperative days 1-5, and was converted into intravenous morphine equivalents for analysis [Grossman 1987]. Our postoperative pain control protocol is as follows: Patients receive intravenous fentanyl after cardiac surgery until extubation. Dexmedetomidine hydrochloride (Precedex, Pfizer, New York, NY, USA) is the primary sedative used during intubation. Following extubation, we use either intravenous morphine or hydromorphone until patient can tolerate oral pain medications. Oral oxycodone or acetaminophen/oxycodone (Percocet) is then prescribed as needed.

Univariate analyses were performed to determine differences in intubation time, ICU stay lengths, hospital stay lengths, subjective pain, and pain medication requirements between patients in Group $C$ and Group R. $P$ values under .05 were considered to be significant in this study.

\section{RESULTS}

During the study period, 416 patients underwent cardiac surgery requiring sternotomy. 288 patients were excluded preoperatively and 48 were excluded intraoperatively as detailed in Figure 1.

Out of a planned 400 patients, a total of 80 patients were randomized and included in this study. 39 patients (49\%) received a rigid closing device, and 41 patients $(51 \%)$ had a conventional wire sternotomy closure. Patient demographics are shown in Table 1. Patients in Group R had a higher body mass index than those patients in Group C (Group R: $31 \pm 5$; Group C: $29 \pm 5 ; P=.04)$. There were no other differences in demographic or preoperative characteristics between the patients in Group C and Group R. There were no significant differences in the percentages of patients in each group who underwent $\mathrm{CABG}$, isolated valve surgery, or combined CABG and valve operations. There were also no significant differences in EuroSCORE between patients in Group C and Group R (Table 1).

Postoperative outcomes for patients are displayed in Table 2. There was no difference between patients in Group $\mathrm{R}$ and Group $\mathrm{C}$ with respect to the primary endpoint of intubation time. Despite trends favoring rigid fixation, there were no significant differences in ICU stay hours, or hospital stay lengths between Group C and Group R. Three patients in Group C had superficial sternal infections, compared to 1 patient in Group R. All of the superficial sternal infections were treated with antibiotics and no patients underwent sternal debridement. One patient in Group $\mathrm{R}$ had a deep sternal infection while no patients in Group $\mathrm{C}$ had this complication. The patient with deep sternal infection was found to have an infection with multiple organisms including drug sensitive Escherichia coli, Klebsiella oxytoca, and Serratia marcescens. This patient was treated with sternal debridement and removal of the sternal plate and subsequent pectorals muscle flap.

There were no differences in pain scores obtained from patients in Group R and Group $\mathrm{C}$ on postoperative days 1 through 5 (Table 2). There was a trend towards less subjective pain in patients in Group $\mathrm{R}$ on postoperative days 3 and 4; however, these results were not statistically significant. 


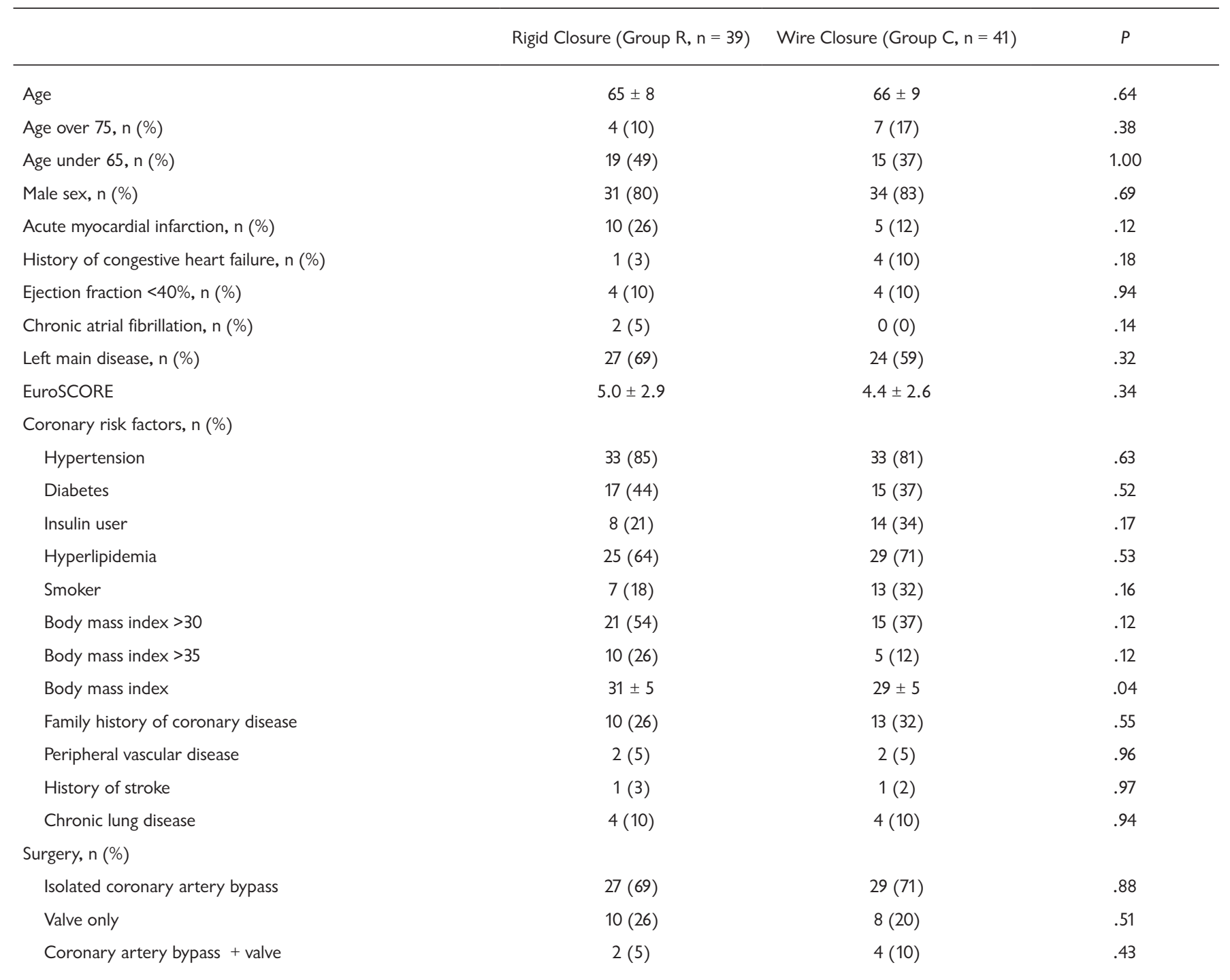

There were also no differences in the intravenous narcotic requirements, or the total narcotic requirements between patients in Group R and Group C on postoperative days 1 through 5. Again, a trend towards requiring lower doses of narcotics was observed for patients in Group R on postoperative day 4, but this result was not significant. No differences were found in the total narcotic requirements over postoperative days 1 to 5 between patients in Group R and Group C. Finally, there was no difference in the last day of narcotics between patients in the two groups (Table 2).

The average number of the SternalockBlu devices per patient was $3 \pm 0.6$, and number of screws per patient was $19 \pm 3$. The average cost of the SternalockBlu devices was $\$ 1261 \pm 209$, as determined by the specific rigid plates used in each patient.

\section{DISCUSSION}

This randomized trial was performed to further investigate results our group obtained from a retrospective analysis showing that rigid sternal fixation was associated with shortened ventilation time, ICU stay, and hospital stay. The present randomized trial showed that in non-high risk patients, there were no significant differences in intubation times when patients undergo rigid sternal fixation as compared to conventional wire sternal fixation. Additionally, no significant differences in short-term pain, ICU stay length, or hospital stay length were observed.

Previous studies have shown distinct benefits to rigid sternal fixation in specific populations. Rigid fixation has been associated with more robust sternal fixation at 3 and 6 months poststernotomy, fewer postoperative complications at 6 months, and a trend towards fewer sternal infections at 6 months postoperation [Raman 2012; Allen 2017]. With this enhanced sternal fixation, usage of a rigid fixation device has also been associated with lower rates of mediastinitis in high-risk populations as compared to wire fixation [Song 2004]. Rigid sternal fixation has also been associated with faster sternal union and lower pain medication usage both 9-12 days and 3 weeks 
Table 2. Results for Patients Undergoing Rigid and Wire Closure

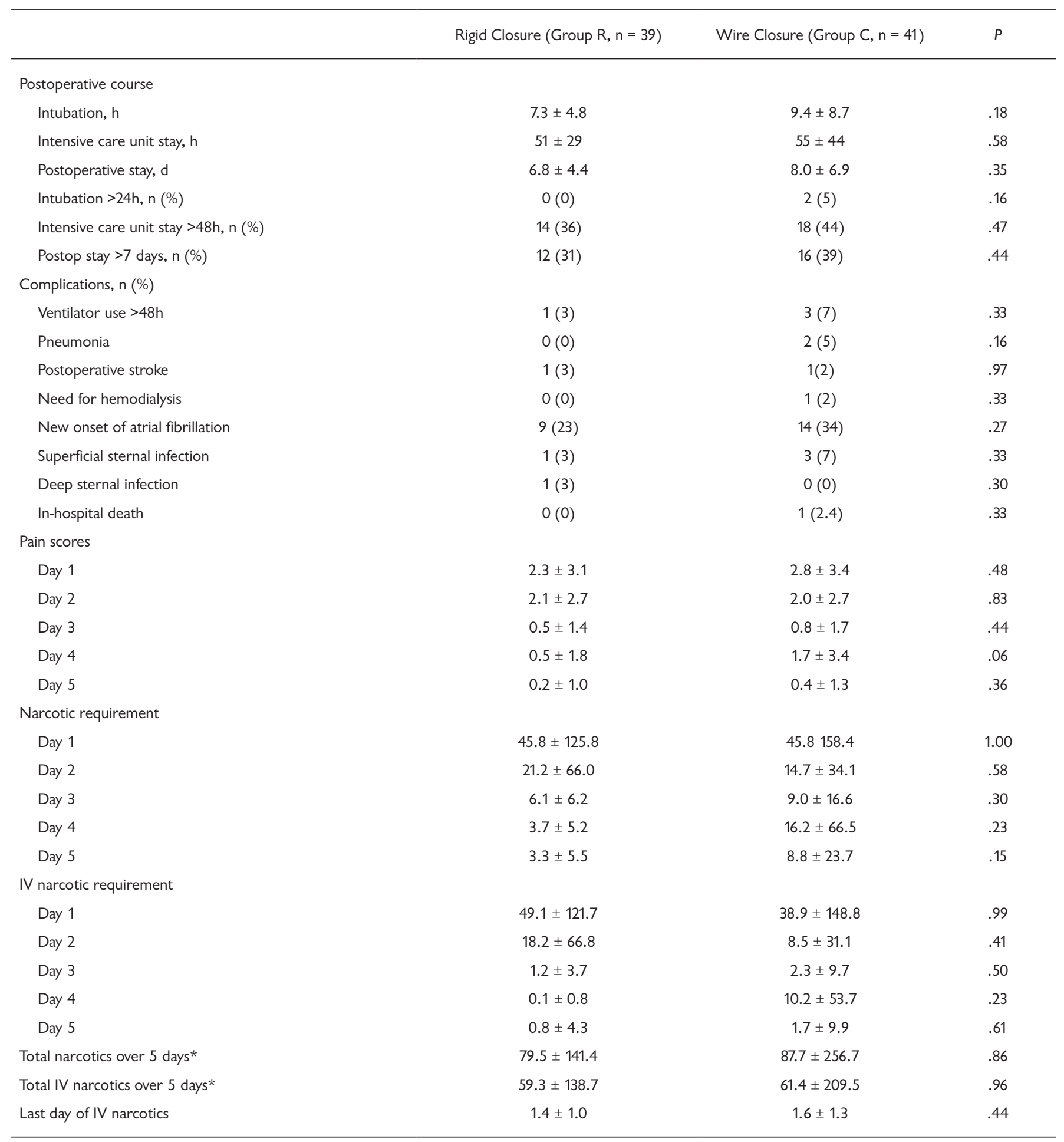

*Narcotic requirements were calculated as intravenous morphine equivalent as explained in the body of the paper.

postoperatively [Raman 2012; Matsuyama 2016]. No previous study has assessed short-term ( $<5$ postoperative days) pain differences in patients who receive rigid and wire sternal closure.
This study was initially designed to investigate a potential difference in intubation time between patients who receive rigid and wire sternal fixation following cardiac surgery. We 
chose intubation time as our primary outcome as we expected the patients in Group R to require fewer narcotics postoperatively, and subsequently regain consciousness sooner than patients in Group C, thus allowing patients in Group R to be extubated earlier. The fact that the intubation time was similar between the two groups may be related to the similar pain medication requirements between patients in Groups R and C.

The results regarding postoperative pain obtained from this study show that the pain reduction benefits of rigid sternal fixation do not begin in the first 5 postoperative days. Other studies have examined the intermediate postoperative period, and show a decrease in pain with rigid fixation. In a group of 64 patients who had either conventional wire or rigid sternal fixation, researchers found a significantly lower pain medication requirement on postoperative days 9-12 among those patients who received rigid sternal fixation [Matsuyama 2016]. Other research has shown decreased pain among patients who receive rigid sternal fixation three weeks postoperatively [Raman 2012]. Based on these results, rigid sternal fixation provides intermediate to long-term pain benefits. Short-term pain reduction with rigid sternal fixation was not demonstrated through our study.

Despite rigid sternal fixation devices being more expensive than wire fixation, a previous cost analysis showed no significant increased costs on the entire hospital stay when using rigid plating systems due to the lower rates of postoperative complications and need for emergent follow-up care [Allen 2017; Park 2017]. The average cost of the SternalockBlu devices used in our patients was $\$ 1261$. This is considerably more expensive than the cost of sternal wires (approximately $\$ 10 /$ wire, 8 wires/patient). If rigid sternal fixation led to a shorter intubation time, ICU stay, or hospital stay to offset the increase in price, one could argue for the use of rigid fixation from a financial perspective. No significant decrease in intubation time, ICU stay, or hospital stay was observed in our study. There were no differences in postoperative complications or the need for emergent followup care in our population. However, despite the results not reaching statistical significance, there were trends towards decreased ICU time and decreased length of stay among those patients who received rigid sternal fixation. Although not statistically significant, the reduced length of hospital stay of 1 day could offset the cost of the sternal plates, and result in a cost-neutral outcome.

As stated above, at initiation of the study, enrollment was intended for 400 patients. Due to the change of our postoperative pain control procedure, and difficulty in enrollment, our study was discontinued after enrollment of 80 patients. While there were trends towards decreased intubation time, ICU time, and hospital length of stay among patients who received rigid sternal fixation, these results did not meet statistical significance. Additionally, because of this small sample size and outlier variables in certain parameters, very high standard deviations were calculated for a number of pain measurements. Despite the trends towards shorter intubation time and lower pain on post-operative days 3 and 4 for patients in Group R, these high standard deviations made reaching statistical significance difficult. A larger sample size is needed to further investigate trends in intubation time and short-term pain with rigid versus conventional wire fixation.

In summary, this randomized trial in a low-risk cardiac surgery population did not show significant differences in intubation time or short-term pain between patients who underwent rigid sternal fixation as compared to sternal wire fixation. No significant differences in ICU stay or hospital stay length were observed for patients receiving rigid sternal fixation, despite trends favoring rigid fixation.

\section{REFERENCES}

Allen KB, Thourani VH, Naka Y, et al. 2017. Randomized, multicenter trial comparing sternotomy closure with rigid plate fixation to wire cerclage. J Thorac Cardiovasc Surg 153:888-96.

Grossman SA, Sheidler VR. 1987. An aid to prescribing narcotics for relief of cancer pain. World Health Forum 8:525-9.

Hirose H, Yamane K, Youdelman BA, Bogar L, Diehl JT. 2011. Rigid sternal fixation improves postoperative recovery. Open Cardiovasc Med J 4:148-52.

Matsuyama K, Kuinose M, Koizumi N, et al. 2016. Sternal closure by rigid plate fixation in off-pump coronary artery bypass grafting: a comparative study. J Artif Organs 19:175-8.

Mozaffarian D, Benjamin EJ, Go AS, et al. 2016. Executive summary: heart disease and stroke statistics-2016 update: a report from the american heart association. Circulation133:447-54.

Nishimura T, Kurihara C, Sakano Y, Kyo S. 2014. Sternalock plating system for elderly post-sternotomy patients. J Artifical Organs 17:288-90.

Ozaki W, Buchman SR, Iannettoni MD, Frankenburg EP. 1998. Biomechanical study of sternal closure using rigid fixation techniques in human cadavers. Annals Thorac Surg 65:1660-5.

Pai S, Gunja NJ, Dupak EL, et al. 2005. In vitro comparison of wire and plate fixation for midline sternotomies. Ann Thorac Surg; 80:962-8.

Park JS, Kuo JH, Young JN, Wong MS. 2017. Rigid sternal fixation versus modified wire technique for poststernotomy closures: a retrospective cost analysis. Ann Plast Surg 78:537-42.

Raman J, Straus D, Song DH. 2007. Rigid plate fixation of the sternum. Ann Thorac Surg 84:1056-1068.

Raman J, Lehmann S, Zehr K, et al. 2012. Sternal closure with rigid plate fixation versus wire closure: a randomized controlled multicenter trial. Ann Thorac Surg 94:1854-61.

Song DH, Lohman RF, Renucci JD, Jeevanandam V, Raman J. 2004. Primary sternal plating in high-risk patients prevents mediastinitis. Euro J Cardiovasc Surg 26:367-72. 